\title{
A LYAPUNOV FUNCTION AND GLOBAL PROPERTIES FOR $S I R$ AND SEIR EPIDEMIOLOGICAL MODELS WITH NONLINEAR INCIDENCE
}

\author{
Andrei Korobeinikov and Philip K. Maini \\ Centre for Mathematical Biology, Mathematical Institute \\ University of Oxford \\ 24-29 St Giles', Oxford, OX1 3LB, UK \\ (Communicated by Yang Kuang)
}

\begin{abstract}
Explicit Lyapunov functions for $S I R$ and $S E I R$ compartmental epidemic models with nonlinear incidence of the form $\beta I^{p} S^{q}$ for the case $p \leq 1$ are constructed. Global stability of the models is thereby established.
\end{abstract}

1. Introduction. It is traditionally postulated that the spread of an infection occurs according to the principle of mass action and associated with it the bilinear incidence rate. However, there are a variety of reasons why this standard bilinear incidence rate may require modification. The incidence rate of the form $\beta I^{p} S^{q}$, were $S$ and $I$ are respectively the number of susceptible and infective individuals in the population (or the fractions of susceptible and infective), and $\beta, p$ and $q$ are positive constants, is the most common nonlinear incidence rate. In recent years models with this incidence rate were considered by several authors, for example Liu et al. [11, 12], Hethcote et al. [6], Hethcote and van den Driessche [7], Derrick and van den Driessche [2, 3], Glendinning and Perry [4], Lizana and Rivero [13] and others. With very few exceptions, these authors focused on the local properties and bifurcations of equilibrium states.

In this note applying the direct Lyapunov method, we consider global properties of $S I R$ and $S E I R$ models with the incidence rate of the form $\beta I^{p} S^{q}$ for the particular case $p \leq 1$. We construct a Lyapunov function which is a generalization of the Lyapunov functions constructed earlier for models with bilinear incidence $[10,8]$. We show that the condition $p \leq 1$ is a sufficient condition for global stability, and that the global properties of the systems do not depend on the value of $q$.

2. Main result. Following the classical assumption [1, 5], we divide a population of a constant size into a number of classes of epidemiological significance denoting respectively by $S, E, I$ and $R$ the fractions of susceptible, exposed, infective and recovered (or removed) individuals in the population, so that $S+(E)+I+R=1$ holds. For the incidence rate of the form $\beta I^{p} S^{q}$, the basic $S I R$ model is

$$
\begin{aligned}
\dot{S} & =b-\beta I^{p} S^{q}-\mu S, \\
\dot{I} & =\beta I^{p} S^{q}-\delta I,
\end{aligned}
$$

2000 Mathematics Subject Classification. 92D30, 34D20.

Key words and phrases. Direct Lyapunov method, endemic equilibrium state, global stability, nonlinear incidence. 
and the basic $S E I R$ model is

$$
\begin{aligned}
\dot{S} & =b-\beta I^{p} S^{q}-\mu S \\
\dot{E} & =\beta I^{p} S^{q}-\sigma E \\
\dot{I} & =\theta E-\delta I
\end{aligned}
$$

Here $b$ is the birth rate, $\mu$ is the susceptible death rate, $\delta$ is the infective removal rate (including the mortality rate) and $\theta$ is the rate with which the exposed population moves into the infective class; $\sigma \geq \theta$ includes also mortality of the exposed individuals. We omit the equations for the recovered population $R$; the constant population size assumption enables us to do so.

If $0<p<1$ holds, then each of these systems has two equilibrium states: an infection-free equilibrium $Q_{0}$ with the coordinates $S_{0}=b / \mu, E_{0}=I_{0}=0$, and an endemic equilibrium state $Q^{*}=\left(S^{*}, E^{*}, I^{*}\right)$, such that

$$
B \delta I^{*}=\beta I^{*^{p}} S^{*^{q}}, \quad \mu S^{*}+B \delta I^{*}=b, \quad \delta I^{*}=\theta E^{*} .
$$

Here $B=\sigma / \theta$ for the $S E I R$ system, or $B=1$ for the $S I R$ system. (In the case $p>1$, these systems have either one, two, or no equilibrium states [11].)

The following Theorem provides global properties of the systems (1) and (2).

TheOREm 2.1. If $p \leq 1$, then the endemic equilibrium states $Q^{*}$ of the models (1) and (2) are globally asymptotically stable. The stability does not depend on the value of the parameter $q$.

Proof. Assume that $p, q \neq 1$. Then for these $S I R$ and $S E I R$ models we construct a Lyapunov function of the form

$$
\begin{aligned}
V(S, E, I)= & A S\left(1+\frac{1}{q-1}\left(\frac{S^{*}}{S}\right)^{q}\right) \\
& +B I\left(1+\frac{1}{p-1}\left(\frac{I^{*}}{I}\right)^{p}\right)+C\left(E-E^{*} \ln E\right) .
\end{aligned}
$$

Here $A=1, B=\sigma / \theta$ and $C=1$ for the $S E I R$ system, or $A=B=1$ and $C=0$ for the $S I R$ system. This function is defined and continuous for all $S, E, I>0$. The function $V(S, E, I)$ satisfies

$$
\frac{\partial V}{\partial S}=A\left(1-\left(\frac{S^{*}}{S}\right)^{q}\right), \quad \frac{\partial V}{\partial E}=C\left(1-\frac{E^{*}}{E}\right), \quad \frac{\partial V}{\partial I}=B\left(1-\left(\frac{I^{*}}{I}\right)^{p}\right)
$$

and hence it is easy to see that $Q^{*}$ is the only extremum and the global minimum of the function in the positive octant $\mathbf{R}_{+}^{3}$. Consequently, the function (4) is indeed a Lyapunov function [14]. This function is a generalization of the Lyapunov functions constructed in $[10,8]$ for the case of the nonlinear incidence rate $\beta I^{p} S^{q}$.

In the case of the SEIR system (2), using the equalities

$$
b=\mu S^{*}+B \delta I^{*}, \quad \beta I^{*^{p}} S^{*^{q}}=B \delta I^{*}, \quad \delta I^{*}=\theta E^{*}, \quad B \theta=\sigma
$$


for the equilibrium state $Q^{*}$, the function $V(S, E, I)$ satisfies

$$
\begin{aligned}
\dot{V}= & b-\beta I^{p} S^{q}-\mu S-b \frac{S^{*^{q}}}{S^{q}}+\beta I^{p} S^{*^{q}}+\mu \frac{S^{*^{q}}}{S^{q-1}} \\
& +\beta I^{p} S^{q}-\sigma E-\beta I^{p} S^{q} \frac{E^{*}}{E}+\sigma E^{*}+B\left(\theta E-\delta I-\theta \frac{I^{*^{p}}}{I^{p}} E+\delta \frac{I^{*^{p}}}{I^{p-1}}\right) \\
= & B \delta I^{*}\left(2-\frac{S^{*^{q}}}{S^{q}}+\frac{I^{p}}{I^{*^{p}}}-\frac{E^{*}}{E} \frac{S^{q} I^{p}}{S^{*^{q}} I^{*^{p}}}-\frac{I}{I^{*}}-\frac{E}{E^{*}} \frac{I^{*^{p}}}{I^{p}}+\left(\frac{I}{I^{*}}\right)^{1-p}\right) \\
& +\mu S^{*}\left(1-\frac{S}{S^{*}}-\frac{S^{*^{q}}}{S^{q}}+\left(\frac{S}{S^{*}}\right)^{1-q}\right) \\
= & -B \delta I^{*}\left(v^{1-p}-1\right)\left(v^{p}-1\right)+B \delta I^{*}\left(3-\frac{1}{u^{q}}-w-\frac{u^{q}}{w}\right) \\
& +\mu S^{*}(1-u)\left(1-\frac{1}{u^{q}}\right) .
\end{aligned}
$$

Here $u=S / S^{*}, v=I / I^{*}$ and $w=E I^{* p} / E^{*} I^{p}$. If $p<1$, then

$$
h(v)=\left(v^{p}-1\right)\left(v^{1-p}-1\right) \geq 0 \text { for all } v>0 .
$$

Since the arithmetical mean is greater than, or equal to the geometrical mean,

$$
g(u, w)=3-\frac{1}{u^{q}}-w-\frac{u^{q}}{w} \leq 0 \text { for all } u, w, q>0
$$

(where the equality holds only if $u=w=1$ ). Furthermore,

$$
(1-u)\left(1-\frac{1}{u^{q}}\right) \leq 0 \text { for all } u, q>0
$$

Therefore, the condition $p<1$ ensures that $\frac{d V}{d t} \leq 0$ for all $S, E, I>0$, where the equality holds only at the equilibrium point $Q^{*}=\left(S^{*}, E^{*}, I^{*}\right)$. By the Lyapunov asymptotic stability theorem [14], the equilibrium point $Q^{*}$ is globally asymptotically stable. This result is valid for the whole positive octant $\mathbf{R}_{+}^{3}$.

If either $p=1$, or $q=1$, then we have to replace in the equation (4) the corresponding term, $\frac{I^{*^{p}}}{p-1} I^{1-p}$, or $\frac{S^{*^{q}}}{q-1} S^{1-q}$ respectively, by the term $-I^{*} \ln I$, or $-S^{*} \ln S$. If $p=q=1$, that is in the case of the bilinear incidence rate $\beta S I$, the function (4) takes the form

$$
V(S, I)=A\left(S-S^{*} \ln S\right)+B\left(I-I^{*} \ln I\right)+C\left(E-E^{*} \ln E\right) .
$$

This Lyapunov function has been constructed for three- and four-compartmental models $[10,8]$ with the standard bilinear incidence.

The case of the SIR system (1) is analogous.

It is obvious that this result does not depend on the value of the parameter $q$.

3. Discussion and conclusion. In this paper we proved global stability of the $S I R$ and $S E I R$ models with the incidence rate $\beta I^{p} S^{q}$. This conclusion is valid under the constant population size assumption, and under the condition $p \leq 1$. In general, this global stability is associated with concavity of the incidence rate with respect to the variable $I$ (see [9] for details). We have to note that in the case $p>1$ the systems can have either one, two, or no equilibrium states, and lose stability $[12,11]$. 
The condition $p \leq 1$ may arise as a consequence of saturation effects: when the number of the infectives is very high, so that exposure to the disease agent is virtually certain, the disease transmission or the incidence rate will respond more slowly than linearly to the increase in $I$. The case of the incidence rate being convex with respect to $I$ (that is the case $p>1$ ) is hardly common: it may arise in some particular cases as a consequence of community effects (we discuss this matter in [9]).

Finally, we point out one particular feature of the incidence rate $\beta I^{p} S^{q}$ : for all $p<1$ the basic reproduction number $R_{0}$ of a model is equal to zero, whereas for $p>1$ it tends to infinity.

Acknowledgments. A. K. is supported by the Foundation for Research, Science and Technology of New Zealand, through Project contract UOXX0101.

\section{REFERENCES}

[1] R.M. Anderson and R.M. May, Infectious Diseases in Humans: Dynamics and Control, Oxford University Press, Oxford, 1991.

[2] W.R. Derrick and P. van den Driessche, emphA disease transmission model in a nonconstant population, J. Math. Biol., 31 (1993), 495-512.

[3] W.R. Derrick and P. van den Driessche, Homoclinic orbits in a disease transmission model with nonlinear incidence and nonconstant population, Discreate and Contin. Dyn. Syst. Ser. B, 3 (2003) 299-309.

[4] P. Glendinning and L.P. Perry, Melnikov analysis of chaos in a simple epidemiological model, J. Math. Biol., 35 (1997), 359-373.

[5] H.W. Hethcote, The Mathematics of infectious diseases, SIAM Rev., 42 (4), (2000), 599-653.

[6] H.W. Hethcote, M.A. Lewis and P. van den Driessche, An epidemiological model with delay and a nonlinear incidence rate, J. Math. Biol., 27 (1989), 49-64.

[7] H.W. Hethcote and P. van den Driessche, Some epidemiological models with nonlinear incidence, J. Math. Biol., 29 (1991), 271-287.

[8] A. Korobeinikov, Lyapunov functions and global properties for SEIR and SEIS epidemic models, Math. Med. Biol. J. IMA, to appear.

[9] A. Korobeinikov and P.K. Maini, Non-linear incidence and stability of infectious disease models, preprint (2004).

[10] A. Korobeinikov and G.C. Wake, Lyapunov functions and global stability for SIR, SIRS and SIS epidemiological models, Appl. Math. Lett., 15 (2002), 955-961.

[11] W.M. Liu, H.W. Hethcote and S.A. Levin, Dynamical behavior of epidemiological models with nonlinear incidence rates, J. Math. Biol., 25 (1987), 359-380.

[12] W.M. Liu, S.A. Levin and Y. Isawa, Influence of nonlinear incidence rates upon the behaviour of SIRS epidemiological models, J. Math. Biol., 23 (1986), 187-204.

[13] M. Lizana and J. Rivero, Multiparametric bifurcations for a model in epidemiology, J. Math. Biol., 35 (1996), 21-36.

[14] A.M. Lyapunov, The General Problem of the Stability of Motion, Taylor \& Francis, London, 1992.

Received on Feb. 11, 2004. Revised on Feb. 15, 2004.

E-mail address: korobein@maths.ox.ac.uk

E-mail address: maini@maths.ox.ac.uk 